\title{
What does IGRA testing add to the diagnosis of ocular tuberculosis? A Bayesian latent class analysis
}

\author{
Rupesh Agrawal ${ }^{1,2,3^{*}}$ (D), Robert Grant ${ }^{4}$, Bhaskar Gupta ${ }^{5}$, Dinesh Visva Gunasekeran³, Julio J. Gonzalez-Lopez ${ }^{1}$,
} Peter K. F. Addison ${ }^{1}$, Mark Westcott ${ }^{1}$ and Carlos E. Pavesio ${ }^{1,2}$

\begin{abstract}
Background: To evaluate the contribution made to the diagnostic work-up for patients with suspected ocular tuberculosis (TB) by QuantiFERON-TB Gold In-Tube (QFT) tests using latent class analysis model.

Methods: A single centre retrospective cohort study. A Bayesian latent class model was constructed on the basis of demographics, phenotypes and test results from patients attending a tertiary referral center in the UK. This estimated the probability of ocular TB for each patient in two versions, first with and then without QFT. The estimated probability of ocular TB was compared with treatment failure.

Results: From a database of 365 patients with clinical signs suggestive of ocular TB, 267 patients who had QFT and complete data were evaluated. Mean age was $45.0 \pm 15.4$ years with 141 (52.9\%) male and 148 (50.5\%) of Asian ethnicity. QFT was positive in 208 (70.1\%) patients and ATT was instituted in 145 (49.5\%) patients with 100 (34.1\%) patients also having concurrent systemic corticosteroid therapy. The best estimate of a QFT level separating TB-positive and TB-negative patients was extremely low. This weak discrimination between TB and non-TB groups was reflected in poor positive and negative predictive values for treatment failure.

Conclusions: The latent class model did not successfully predict treatment failure, despite taking all variables into account. The threshold between TB and non-TB in QFT values was implausibly low and removing QFT from the model made prediction slightly worse. A larger prospective study is required to establish the role of all tests, demographics and phenotypes in diagnosis.
\end{abstract}

Keywords: Extrapulmonary latent TB, Presumed ocular tuberculosis, Positive QFT, ATT, Uveitis, Bayesian latent class analysis

\section{Background}

Tuberculosis (TB) still remains a major public health problem in most countries, including developed nations. With a large number of immigrants in the United Kingdom (UK), TB is disproportionately distributed amongst new migrants and certain ethnic groups who carry social risk factors and tend to be more adversely affected. [1] Seven thousand, eight hundred and ninety-two cases of TB were notified in the UK in 2013 with an incidence of $12.3 / 100,000$. London accounted for the highest proportion

\footnotetext{
* Correspondence: rupeshttsh@gmail.com

'Moorfields Eye Hospital, NHS Foundation Trust, London, UK

${ }^{2}$ Biomedical Research Centre, UCL Institute of Ophthalmology, London, UK

Full list of author information is available at the end of the article
}

of cases in the UK $(37.8 \%, 2985 / 7892)$, with a rate of 35.5/ 100,000. [1] A significant proportion of these patients presents with extrapulmonary latent mycobacterium TB. Over the last decade, we have witnessed an apparent increase in the incidence of ocular TB. [1] This apparent increase may be attributable to a higher rate of diagnosis due to availability of the interferon gamma release assay test (IGRA). Ocular TB, as a form of latent extrapulmonary $\mathrm{TB}$ is faced with the multifaceted problem of diagnosis from inconclusive signs and corraborative investigations. It can present as anterior uveitis, intermediate uveitis, vitritis, retinal vasculitis, neuroretinitis, solitary or multiple choroidal tubercles, serpiginous-like choroiditis, subretinal abscess, endophthalmitis and panophthalmitis [2-6]. 
Ocular TB has been postulated to arise either as a result of direct infection with TB bacilli or as a hypersensitivity reaction to latent TB infection. [4, 6-8]. Invitro assessment of interferon-gamma (IFN $\gamma$ ) via IGRA has been an important addition to the diagnosis of TB and has increased the sensitivity in diagnosing latent TB. $[9,10]$ QuantiFERON-TB Gold In-tube test (QFT; Cellestis Limited, Carnegie, Victoria, Australia) is Food and Drug Administration (FDA) approved IGRA test used for identifying latent TB infection. [9-11] According to the manufacturer's instructions, QFT is considered positive for values $>0.35 \mathrm{IU} / \mathrm{mL}$. Based on Centers for disease control and prevention (CDC), QFT was approved to be used for diagnosis of latent TB globally and same was adopted by National Institute for Health and Care Excellence (NICE) in the UK. [12].

The absolute values of QFT was interrogated by Gineys et al. and values of $2 \mathrm{IU} / \mathrm{ml}$ was suggested to be associated with better outcome in patients with ocular TB from low endemic settings. [13] Positive QFT results can occur in patients with non uveitic entities (optic neuritis/ orbital inflammation/ myositis/ sarcoidosis) and also in patients with non tuberculous bacilli (M. Kansasii, M. szulgai, M. marinum) or even on false handling of samples (with erroneously large number of TB antigens in QFT tube) leading to lack of specificity of the test. [14, 15] The reported specificity of the QFT for pulmonary and latent TB ranges from 91 to $99 \%$, but reported sensitivity is somewhat lower $89-91 \%$. [16, 17] The accuracy of IGRA in patients with ocular TB in a BCG-vaccinated, non-endemic population was recently evaluated and reported sensitivity and specificity were 80 and $85 \%$, signifying impact of IGRA in this cohort of population. [18] In a recent review published by Getahun et al., the authors have looked into the worldwide literature and concluded that there is no perfect method for the diagnosis of latent mycobacterial infection [19].

The term "presumed ocular TB" is more commonly used, where no confirmatory ocular investigation is available, but the overall clinical picture, be it via systemic investigations or response to anti-tubercular therapy (ATT), makes the presumptive diagnosis of ocular TB likely. Given the lack of confirmation of local infection, this brings us to the fundamental question of whether a "presumed ocular TB" is truly an ocular TB, and which investigations are sensitive and specific enough to aid in improving the accuracy of our diagnosis. Also, the presence of a significantly broad range of positive values coupled with a myriad of clinical presentations has raised some critical questions:

- Is presumed ocular TB truly ocular TB?

- Is there any association between level of QFT positivity and likelihood of response to ATT [13].
- Do QFT values associate with any pre-treatment clinical features that might predict response to ATT in cases of suspected ocular TB, and if so, is there a threshold value? [13]

Rosenbaum et al. has described in their study on utility of routine screening of patients with uveitis for TB, post-test probability of a test can be deduced, via Bayes' theorem, by using the known sensitivity and specificity of test as well as pre-test probability of the presence of ocular TB. [20] In the present study, we will investigate the predictive value of the QFT for the diagnosis of presumed ocular TB using a Bayesian latent class analysis of the diverse population in a tertiary eye care setting, so as to determine if QFT is justifiable as a routine screening tool for patients with presumed ocular TB. Our aims were to combine all available data into a statistical model predicting each patient's ocular TB status, and to compare the ability of this model, with and without the QFT results, to predict treatment failure (steroid taper, defined below).

\section{Methods}

This was a retrospective cohort study of patients seen at a large tertiary referral centre in the UK. Medical records of patients with presumed ocular TB were reviewed following approval by the Institutional Review Board (IRB). Patient records/information was anonymized and de-identified prior to analysis. This research was performed in adherence to the tenets of the declaration of Helsinki. Records of all the cases between 2005 to 2013 who had IGRA test and Mantoux test done were identified based on data from clinical laboratory at the centre.

The clinical case definition of presumed ocular TB was based on broad non-specific clinical signs proposed by Gupta et al. in 2007. [4] Clinical phenotypes suggestive of TB include anterior uveitis (granulomatous, nongranulomatous), intermediate uveitis, posterior uveitis (choroidal tubercle or tuberculoma, subretinal abscess, serpiginous-like choroiditis), panuveitis, retinitis, retinal vasculitis and neuroretinitis. Patient data were retrieved and data collected included information on demographics, morphological diagnosis, investigations and therapeutic regimens. Patients at presentation were examined by the residents, along with senior uveitis specialists, for signs suggestive of ocular TB. All patients underwent comprehensive examination and investigations to exclude other infectious diseases and immunologic disorders, including chest radiograph. Angiotensin converting enzyme (ACE) and serology tests (HIV, syphilis, hepatitis $\mathrm{B}$ and $\mathrm{C}$ ) were done, if deemed necessary. Patients with a clinical diagnosis of uveitis suggestive of TB in one or both eyes were included in the study. The QFT assay was 
performed as per manufacturer's recommendations and results were interpreted as per the recommendations from manufacturer. [21] Patients with one of the ocular phenotypes mentioned above, and an elevated QFT or other features suggestive of ocular TB, were referred to a respiratory physician for initiation of ATT. All attempts were made to exclude cases with sarcoidosis using information from combination of biochemical test or radiological test.

ATT was not considered in:

- Patients who had previously completed a full course of ATT within the last 10 years as recurrence in these cases was considered improbable. ${ }^{10}$ In this context, a positive QFT result was considered as merely immunologic memory. The authors relied on the history as notified by the patient or patient medical summary from their general practitioners, if available. No separate effort was made to collect past medical history.

- Patients who had mild anterior uveitis or retinal vasculitis, without visual loss. This is because of the frequency of unacceptable severe adverse effects of ATT as compared to the expected potential of ocular benefit [10].

Moreover, patients with insufficient follow-up visits were excluded from the study. Data were subsequently double-entered into a database by two co-authors (RA and JC) and cross-checked for accuracy; discrepancies resolved by referring back to medical records.

\section{Definition of outcomes}

Treatment failure was defined in patients on ATT as:

- Inability to taper oral corticosteroids to less than $10 \mathrm{mg} /$ day or topical steroids to less than two times per day,

- Inability to stop oral immunosuppressive agent, or

- Persistence or recurrence of inflammation within first 6 months of completion of ATT.

For patients not on ATT, failure was defined as: (1) or (2) as stated above respectively, or: persistence or recurrence of inflammation despite treatment for at least 12 months.

\section{Statistical analysis}

In line with manufacturer's recommendations, values of QFT $<0.35$ units were regarded as being equivalent to zero. A transformation of the QFT data was sought to facilitate model a mixture of small, positively skewed values (representing TB-negative patients) and larger, symmetrically-distributed values (representing TBpositive patients) [22].
Exploratory analyses were conducted to find differences in the use of ATT and treatment failure, as well as QFT or ACE between ethnic groups, gender, or presence/absence of binary signs, using non-parametric statistical tests. Correlation between age and QFT, as well as ACE and QFT, was assessed using Spearman's rho coefficient. All exploratory analyses were conducted in R software (version 3.2.3) [www.r-project.org]. Raised ACE may signify a sarcoid kind of presentation and as per our recently published classification. [23] It represents tuberculous-sarcoid kind of presentation and not purely a tubercular type of presentation [23].

A Bayesian latent class model was then constructed using Stan software (rstan interface version 2.8.0) [mc-stan.org]; code is given in the technical appendix (Additional file 1). Bayesian methods are useful for statistical modelling in the context of our data because they can accommodate complex models and provide intuitive outputs regarding values of interest in terms of probability. They are solved by computer-intensive simulation to find values that fit the observed data. [24] (Additional file 1). Statistically, the estimated patient status is called a latent class, but to avoid confusion between latent class and latent TB infection, we use the term "estimated probability of ocular TB". All associations in the model are shown together in Fig. 1. The latent class approach allows for relationships that both predict the estimated probability of ocular TB (for example, ethnicity is a proxy risk factor for exposure to Mycobacterium Tuberculosis), and are a result of, ocular TB (for example, the risk of choroiditis might differ between the patients who truly have ocular TB and those who do not). For each relationship there was a regression coefficient, and all the relationships are linked and evaluated simultaneously, along with each patient's estimated probability of ocular TB. This approach is increasingly popular to evaluate diagnostic tests in the absence of a gold standard [25, 26].

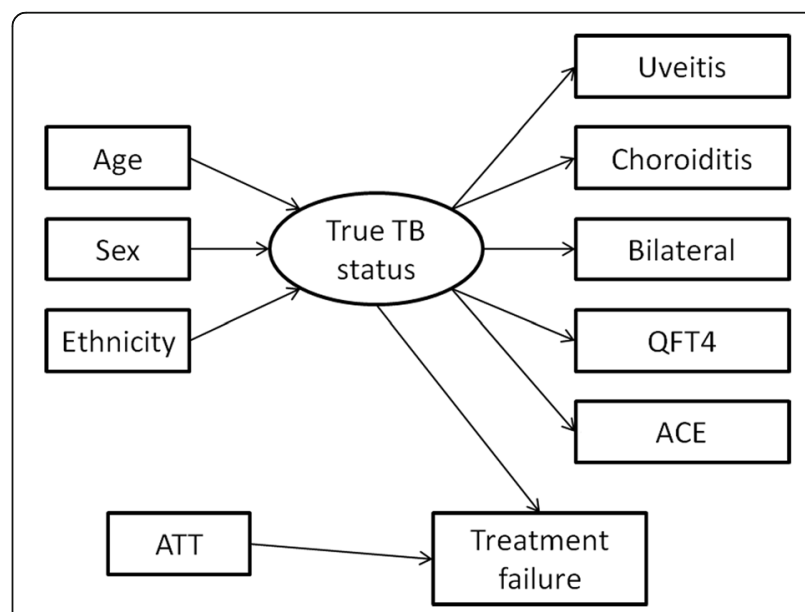

Fig. 1 Latent model of presumed ocular tuberculosis with all possible associations. Abbreviations: TB - Tuberculosis, $\boldsymbol{A} \boldsymbol{T}$ - Antitubercular therapy 
We did not include subjective opinions as prior probabilities, but instead used the weakly informative prior approach advocated by Gelman and colleagues, [26] which avoids extreme values that are implausible and potentially problematic for computation. Residual QFT values (the mean of the observed QFT minus predicted QFT, across all steps from Stan) were plotted against observed values and other potential predictors such as chest X-ray evidence of prior active pulmonary TB to diagnose potential problems in the models. Each patient's estimated probability of ocular TB was plotted with its confidence interval and against the recorded level of QFT to highlight strengths and weaknesses of the model. The dataset is provided in Additional file 2.

\section{Results}

Out of a total of 365 patients with clinical signs suggestive of ocular TB, 298 patients had QFT tests, of whom 267 had complete data in all required variables for the Bayesian model. Mean age was 45.0 years with standard deviation 15.3, 141 (52.8\%) were male, 141 (52.8\%) were of Asian ethnicity, 55 (20.6\%) African and 71 (26.6\%) Caucasian. QFT was recorded as zero for 30 patients and as " $<0.35$ " for another six, who were counted as zero for this analysis; the mean QFT was 5.1 with standard deviation 6.3 (median 2.0, inter-quartile range (IQR) 0.1 to 8.8). Both eyes were involved in 162 (60.7\%) patients. There was presence of anterior uveitis in 49
(18.4\%) patients, intermediate in $65(24.3 \%)$ patients, posterior in $95(35.6 \%)$ patients. Choroidal involvement was present in $34(12.7 \%)$ patients. $58(21.7 \%)$ patients had uveitis at more than one locations with features either suggestive of panuveitis or retinal vasculitis and/ or choroiditis with vitritis and anterior chamber reaction. Evidence of signs suggestive of previous pulmonary disease was present on chest X-ray for 51 patients (19.1\%). The mean ACE was 37.5 with standard deviation 27.9 (median 31, IQR 22 to 44). ATT was instituted in $130(48.7 \%)$ patients with 91 (34.1\%) patients having concurrent systemic corticosteroid therapy. ATT was most commonly given for 6 months $(36.2 \%, 47 / 130)$ or 12 months $(33.84 \%, 44 / 130)$. Descriptive statistics are shown in Table 1.

The 58 (21.7\%) patients with panuveitis features (with uveitis at more than one location) appeared differed significantly from those with uveitis in the following ways:

- more of them had QFT values over the manufacturer's threshold of 0.35 (48/58 [82.8\%] compared to 143/209 [68.4\%], $p=0.048$ by chi-squared test)

- they are younger (median 40 compared to 44 , $p=0.048$ by Wilcoxon-Mann-Whitney test)

- more of them had choroiditis (15/58 [25.9\%] compared to $19 / 209$ [9.1\%], $p=0.002$ by chisquared test)

Table 1 ATT use and treatment failure

\begin{tabular}{|c|c|c|c|}
\hline & ATT given & ATT and tx failure & No ATT and tx failure \\
\hline Male & $61.7 \%(87 / 141)$ & $16.1 \%(14 / 87)$ & $29.6 \%(16 / 54)$ \\
\hline Female & $34.1 \%(43 / 126)$ & $34.9 \%(15 / 43)$ & $36.1 \%(30 / 83)$ \\
\hline Asian & $50.4 \%(71 / 141)$ & $23.9 \%(17 / 71)$ & $31.4 \%(22 / 70)$ \\
\hline African & $49.1 \%(27 / 55)$ & $18.5 \%(5 / 27)$ & $50.0 \%(14 / 28)$ \\
\hline Caucasian & $45.1 \%(32 / 71)$ & $21.9 \%(7 / 32)$ & $25.6 \%(10 / 39)$ \\
\hline Oral steroids & $60.7 \%(91 / 150)$ & $26.4 \%(24 / 91)$ & $50.8 \%(30 / 59)$ \\
\hline No oral steroids & $33.3 \%(39 / 117)$ & $12.8 \%(5 / 39)$ & $20.5 \%(16 / 78)$ \\
\hline No choroidal involvement & $48.5 \%(113 / 233)$ & $22.1 \%(25 / 113)$ & $32.5 \%(39 / 120)$ \\
\hline Choroidal involvement & $50.0 \%(17 / 34)$ & $23.5 \%(4 / 17)$ & $41.2 \%(7 / 17)$ \\
\hline Anterior uveitis & $44.9 \%(22 / 49)$ & $22.7 \%(5 / 22)$ & $33.3 \%(9 / 27)$ \\
\hline Intermediate uveitis & $44.6 \%(29 / 65)$ & $24.1 \%(7 / 29)$ & $27.8 \%(10 / 36)$ \\
\hline Posterior uveitis & $52.6 \%(50 / 95)$ & $20.0 \%(10 / 50)$ & $51.1 \%(23 / 45)$ \\
\hline Posterior uveitis with choroidal involvement & $47.4 \%(9 / 19)$ & $11.1 \%(1 / 9)$ & $60.0 \%(6 / 10)$ \\
\hline Unilateral involvement & $50.5 \%(53 / 105)$ & $18.9 \%(10 / 53)$ & $34.6 \%(18 / 52)$ \\
\hline Bilateral involvement & $47.5 \%(77 / 162)$ & $24.7 \%(19 / 77)$ & $32.9 \%(28 / 85)$ \\
\hline QFT $\leq 0.35$ & $46.1 \%(35 / 76)$ & $31.4 \%(11 / 35)$ & $24.4 \%(10 / 41)$ \\
\hline QFT $0.35-2.00$ & $47.4 \%(27 / 57)$ & $25.9 \%(7 / 27)$ & $43.3 \%(13 / 30)$ \\
\hline QFT $>2.00$ & $50.7 \%(68 / 134)$ & $16.2 \%(11 / 68)$ & $34.8 \%(23 / 66)$ \\
\hline All patients & $48.7 \%(130 / 267)$ & $22.3 \%$ (29/130) & $33.6 \%(46 / 137)$ \\
\hline
\end{tabular}

QFT QuantiFERON -TB Gold In-Tube test, ATT Antitubercular therapy 
- they had higher ACE levels (median 34 compared to 30, $p=0.02$ by Wilcoxon-Mann-Whitney test)

- fewer of them had bilateral symptoms $(27 / 58$ [46.6\%] compared to $135 / 209$ [64.6\%], $p=0.02$ by chi-squared test)

Exploratory analyses revealed that QFT values were significantly lower in patients of Caucasian ethnicity $(p=0.0007)$, in patients not receiving oral steroids $(p=0.03)$, patients with choroiditis $(p=0.04)$, and in the presence of chest X-ray suggestive of healed pulmonary disease $(p=0.04)$. Levels of ACE (Table 1$)$ was higher in patients with choroidal involvement (mean 45.8, median 38.5) than those without (mean 36.3, median 31.0), with $p=0.01$. In Table 2, we show the use of ATT and the proportion with treatment failure broken down by characteristics. The striking feature is that treatment failure is higher in those not treated with ATT than those treated, for all categories except the very lowest values of QFT, which suggests that there are some false negatives in the diagnosis. We cannot estimate the false positives with the information available.

Raising QFT to the power of 0.25 (the 'quartic root') produced a distribution amenable to further statistical modelling (Fig. 2); we refer to this as QFT4. It can be seen to fall into two parts, one positively skewed with mode close to zero, and the other symmetric and centred near $1.2(\mathrm{QFT}=5.28)$. The higher part appears bimodal (has two peaks) in this chart but with relatively small numbers, and in the absence of a biological explanation, we regard this as one common group of patients. Future research with larger samples can confirm the distribution of QFT values in this population. The least common region, which separates these two distributions and is the best candidate for a clinical cut-off level, is near 0.4 (QFT $=0.25)$.

Relationships between the data and the estimated probability of ocular TB are shown in Table 3 . This table shows, firstly, the effect of the patient characteristics on the estimated probability of ocular TB. The uncertainty in these values is represented by the wide confidence intervals (CI), and there are no significant associations, although the direction of effect is in agreement with Table 2. Secondly, the effect of the estimated probability of ocular TB on various signs and symptoms is shown, where the coefficients either represent mean differences or log-odds ratios. Posterior uveitis was significantly more common in patients likely to have TB, while anterior and intermediate uveitis was significantly less common. There was no significant difference in bilateral versus unilateral involvement. Effects on ACE and choroiditis were very imprecise, most likely for reasons of uncertainty addressed below. Finally, the effect of ATT on treatment failure is shown, and although ATT's effect in the absence of TB is not significant, there is a significant reduction in treatment failure when TB is present, as defined by an estimated probability of ocular TB over $50 \%$. All the patients who had previous ATT $(n=8)$ were assessed by a respiratory physician, if there was a risk of reinfection in this high-risk population. However,

Table 2 QuantiFERON -TB Gold In-Tube test (QFT) values obtained in different groups of patients

\begin{tabular}{llll}
\hline Characteristics & Categories & Mean (SD) & Median (IQR) \\
\hline Sex & Male $(n=141)$ & $5.66(6.65)$ & $2.62(0.58-9.05)$ \\
Ethnicity & Female $(n=126)$ & $4.55(5.94)$ & $1.54(0.04-7.86)$ \\
& Asian $(n=141)$ & $5.50(5.89)$ & $2.65(0.79-9.05)$ \\
& African $(n=55)$ & $5.76(6.54)$ & $3.19(0.08-10.40)$ \\
Oral steroids & Caucasian $(n=71)$ & $3.92(6.94)$ & $0.70(0.01-4.45)$ \\
& No $(n=117)$ & $4.61(6.54)$ & $1.49(0.02-8.00)$ \\
Choroiditis & Yes $(n=150)$ & $5.55(6.17)$ & $2.47(0.59-9.86)$ \\
& Absent $(n=233)$ & $4.98(6.35)$ & $1.72(0.05-8.76)$ \\
Uveitis & Present $(n=34)$ & $6.22(6.22)$ & $4.01(1.65-8.86)$ \\
& Anterior $(n=49)$ & $4.83(5.93)$ & $1.39(0.10-8.89)$ \\
Bilaterality & Intermediate $(n=65)$ & $5.61(7.50)$ & $1.62(0.03-10.01)$ \\
& Posterior $(n=95)$ & $4.84(5.96)$ & $1.84(0.05-8.26)$ \\
Chest X-ray suggestive of healed pulmonary TB & Unilateral $(n=105)$ & $4.83(6.10)$ & $1.51(0.27-8.57)$ \\
Total $(n=267)$ & Bilateral $(n=162)$ & $5.34(6.50)$ & $2.59(0.04-8.90)$ \\
\hline
\end{tabular}

SD Standard deviation, IQR Interquartile range 

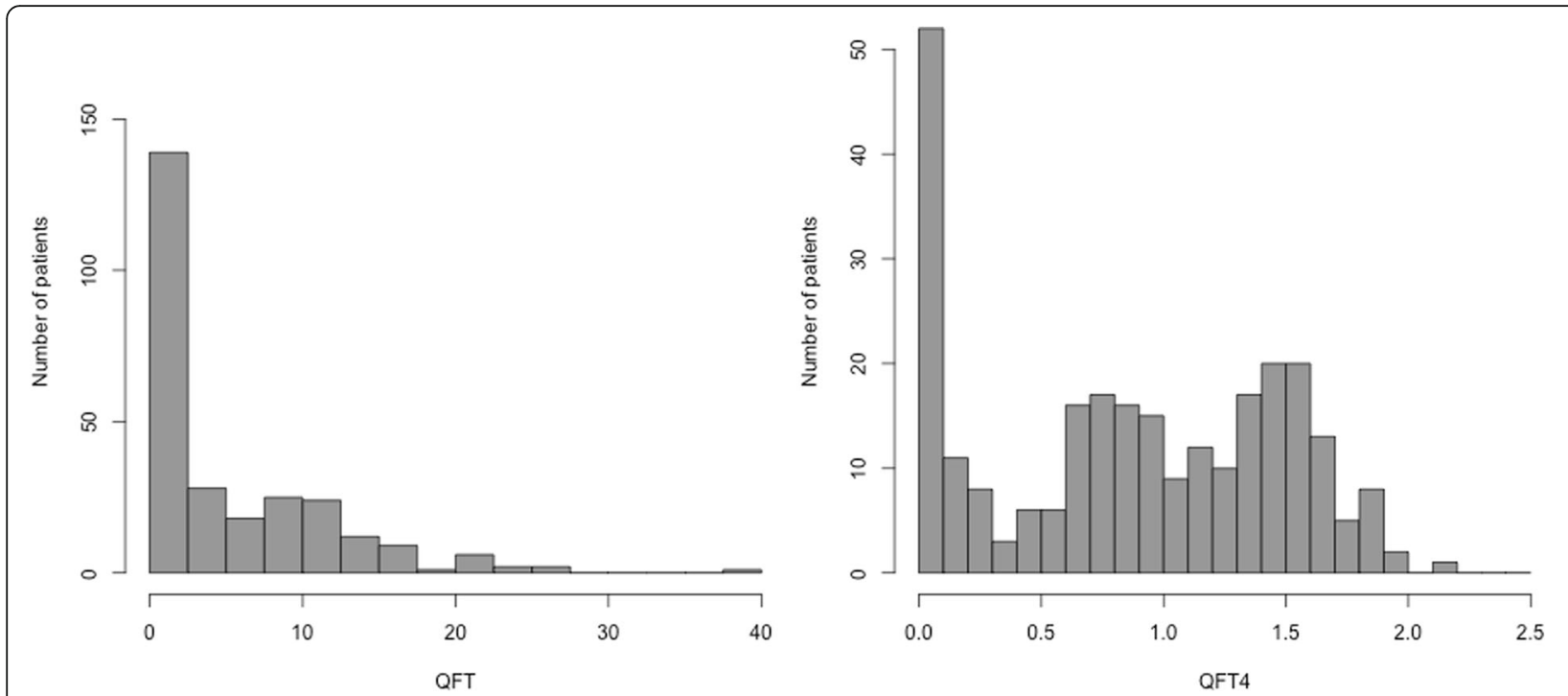

Fig. 2 Histograms of QFT values (left) and QFT4 transformed by raising QFT to the power of 0.25 (right). Abbreviations: QFT - QuantiFERON Gold In-Tube

in absence of the retreatment, they did not demonstrated any higher risk of relapse or treatment failure. Thirty eight patients with mild or non sight threatening disease did not had ATT based on discretion of treating ophthalmologist and respiratory physician.

The model does not provide satisfactory predictive ability. Each patient's estimated probability of ocular TB, with 95\% confidence intervals, is shown in Fig. 3. Of the 267 patients, 68 had CIs straddling either side of 0.5, while 47 were entirely below and 152 entirely above. Based on the estimated probability, taking 0.5 as a cutoff, 68 were below 0.5 and 199 above, which might be regarded as probably not $\mathrm{TB}$ and probably $\mathrm{TB}$ respectively. However, this classification corresponds poorly to

Table 3 Parameters from latent class model

\begin{tabular}{|c|c|c|}
\hline & Estimated effect & $95 \% \mathrm{Cl}$ \\
\hline \multicolumn{3}{|l|}{ Equation estimating the probability of ocular TB } \\
\hline Asian ethnicity ${ }^{a}$ & 1.33 & 0.94 to 1.89 \\
\hline African ethnicity $^{a}$ & 1.13 & 0.76 to 1.68 \\
\hline Female $^{a}$ & 0.89 & 0.64 to 1.24 \\
\hline Age (10 year increment, centred on 40$)^{a}$ & 1.08 & 0.87 to 1.37 \\
\hline \multicolumn{3}{|c|}{ Equations predicting observed signs and symptoms based on the probability of ocular TB } \\
\hline Mean QFT4 if TB+ & 1.19 & 1.12 to 1.26 \\
\hline Mean QFT if TB+ & 5.15 & 4.30 to 6.19 \\
\hline SD of QFT4 if TB+ & 0.44 & 0.39 to 0.49 \\
\hline SD of QFT4 if TB- & 0.06 & 0.05 to 0.07 \\
\hline Crossover between TB+ and TB- QFT distribution (not transformed to QFT4) & 0.05 & 0.04 to 0.07 \\
\hline Effect of estimated probability of ocular TB on anterior uveitis symptoms ${ }^{b}$ & 0.69 & 0.45 to 1.02 \\
\hline Effect of estimated probability of ocular TB on intermediate uveitis symptoms ${ }^{b}$ & 0.55 & 0.36 to 0.82 \\
\hline Effect of estimated probability of ocular TB on posterior uveitis symptoms ${ }^{b}$ & 1.54 & 1.09 to 2.18 \\
\hline Effect of estimated probability of ocular TB on choroiditis ${ }^{b}$ & 21,246 & 0.97 to 3.10 \\
\hline Effect of estimated probability of ocular TB on bilateral symptoms ${ }^{\mathrm{b}}$ & 1.04 & 0.75 to 1.46 \\
\hline Effect of estimated probability of ocular $\mathrm{TB}$ on $\mathrm{ACE}^{\mathrm{C}}$ & 3.99 & -0.40 to 8.23 \\
\hline
\end{tabular}

TB Tuberculosis, QFT QuantiFERON Gold In-Tube, ATT Anti-tubercular therapy, SD Standard deviation, CI Confidence interval

${ }^{a}$ This can be interpreted as an odds ratio for this characteristic in patients with tuberculous uveitis compared to those without

${ }^{\mathrm{b}}$ This can be interpreted as the odds ratio for having a particular sign or symptom if the patient has tuberculous uveitis (predicted probability $>50 \%$ ), compared to not

${ }^{\mathrm{c}}$ This can be interpreted as an additional odds ratio that is applied to the prediction of treatment failure 
treatment failure, which we expected in those who had $\mathrm{TB}$ and were not treated with ATT, or did not have TB but were treated with ATT. The positive predictive value (PPV) was $58.7 \%$ and the negative predictive value $56.1 \%$, which is not much better than chance.

When the model is run again without QFT, the confidence intervals of Fig. 3 are much wider, with 259 patients straddling $0.5,8$ with CIs entirely below, and none entirely above. The estimated probability was above 0.5 for 148 patients and below for 119, and from this prediction, the PPV was $51.4 \%$ and NPV $40.3 \%$.

Despite the means of the two-part distribution being well represented by the estimates in Table 3, the standard deviations are very wide. The estimated point of crossover between the distribution of TB-negative and -positive patients was at QFT $=0.05$ with $95 \%$ CI 0.04 to 0.07 . However, as seen in Fig. 4, the relationship between QFT4 and the estimated probability of ocular TB (which is informed in part by QFT4 itself) breaks down at high probabilities.

\section{Discussion}

Currently there is no clear consensus on the diagnosis of ocular TB and this remains a challenge for clinicians around the world. There are many postulations behind the pathophysiology of ocular TB. It may be an immune mediated ocular inflammation that is initially triggered by mycobacterium $\mathrm{TB}$, without any underlying active infection. [27] Alternatively, it may result from an inoculum directly within the eye. [28] Although such direct TB infection may manifest such as a choroidal abscess, the majority of patients displayed clinical features, which were not exclusively associated with TB infection. None of the clinical phenotypes in our series from the low endemic region showed a statistically significant association for reduced risk of recurrence with positive or better response to ATT. In our current study, we have investigated the possible predictive value of QFT for the diagnosis of presumed ocular TB from a cohort of patients with signs strongly suggestive of ocular TB, based on Bayesian model analysis.

The best estimate of a QFT level separating TB-positive and TB-negative patients is extremely low, leading most likely to high sensitivity but poor specificity. Although it is not inconsistent with the least common region seen in the observed data around 0.23 , the uncertainty is too great to permit a firm conclusion separating the patients into two groups. This indicates the considerable uncertainty about true TB status, even taking all variables into account in a predictive model. In our current model, patients with higher QFT levels and treated with ATT had lower treatment failure as against those patients who did not had ATT with lower QFT values.

Ang et al. have earlier researched the usefulness of IGRA in diagnosis of presumed ocular TB, however the authors have not specified the clinical phenotype associated with positive IGRA and have instead enlisted the anatomic location of uveitis [17]. They have reported a poor sensitivity (36\%) and modest specificity $(75 \%)$ of IGRA test alone in presumed tuberculous uveitis; the area under the receiver-operator curve (AUC: a measure

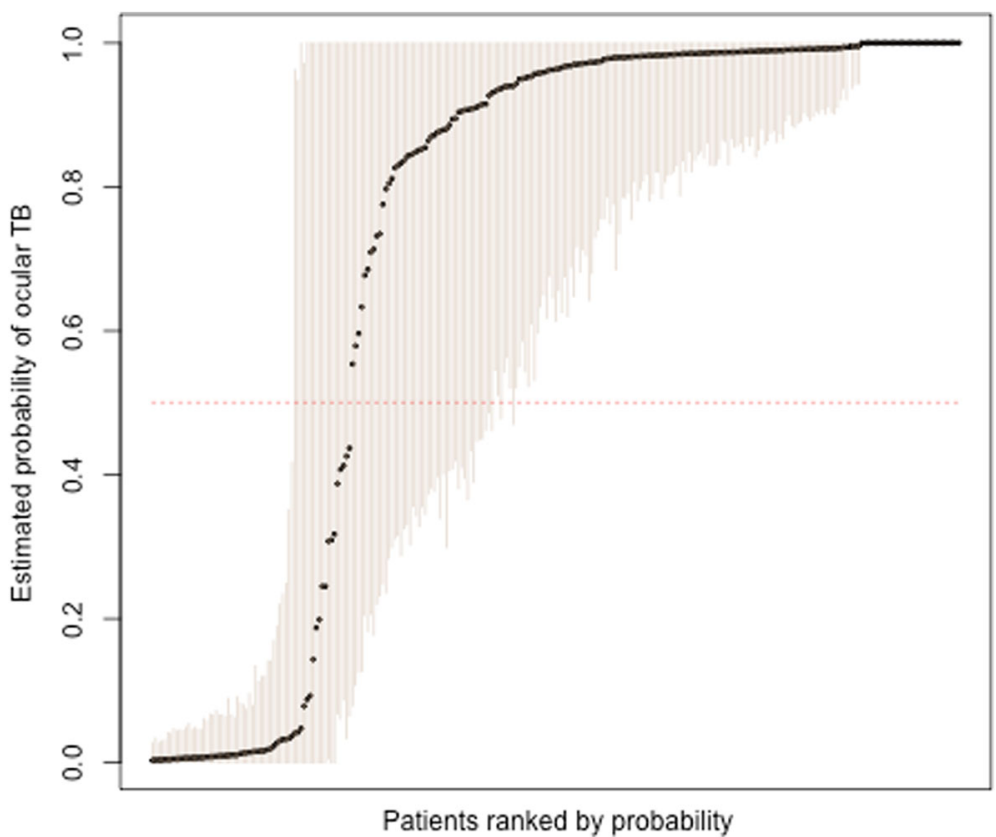

Fig. 3 Patients' estimated probabilities of ocular TB (black dots), ranked from lowest to highest, with 95\% confidence intervals (faint vertical lines). $50 \%$ probability is shown as a horizontal dotted line 


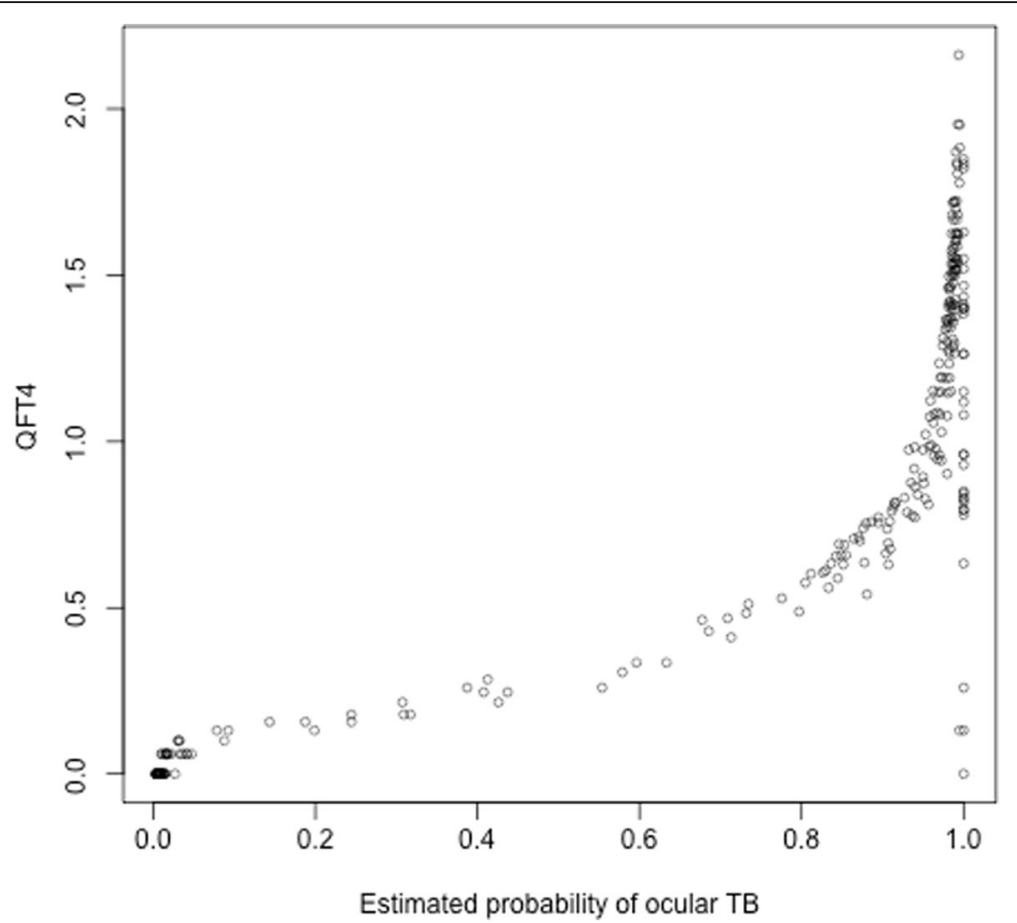

Fig. 4 Patients' estimated probabilities of ocular TB, plotted against the observed QFT4. A good predictive association would show as clusters of dots in the top right and bottom left parts of the plot

of diagnostic ability combining sensitivity and specificity that ranges from 0 to 1 ) of 0.555 . Combining the IGRA with the results of tuberculin skin testing improved the performance somewhat (AUC of 0.644). The authors concluded that both IGRA and tuberculin skin test had poor sensitivities and that neither test could reliably exclude ocular TB if there were highly suggestive clinical features [17].

Rather than assume a gold standard and use ROC analysis, we have taken a Bayesian approach, as this allows us to express uncertainty around each patient's estimated probability of ocular TB. Our data suggest that QFT alone does not adequately separate $\mathrm{TB}$ uveitis from non-TB uveitis. There are very limited studies on the possible predictive clinical signs from low endemic regions, further compounding the diagnostic conundrum. The challenge may be considered greater in countries such as the UK in which TB is much less prevalent but have a very high number of immigrant population from high endemic zones.

La Distia Nora et al. evaluated the clinical manifestations of patients with intraocular inflammation with positive QFT in nonendemic settings. [29] In the series of 77 patients, $85 \%$ of patients were found to be immigrants from endemic region. Retinal occlusive vasculitis and serpiginous choroiditis were commonly noted phenotypes with mean QFT value of $7.5 \mathrm{IU} / \mathrm{ml}$. Like the current study, the authors did not find any positive association between QFT positivity and clinical signs. The authors and the editorial from Pepple et al. have concluded the limited role and risk of false positivity in patients with signs suggestive of ocular TB in low endemic settings and the raised QFT may albeit represent sarcoidosis. $[29,30]$ To further investigate the association between TB and sarcoidosis, we did correlation analysis between potential biomarkers of sarcoidosis (serum ACE) and TB (QFT). In our study we found no statistically significant association between raised serum ACE and QFT.

In another series by Ahn et al. from Korea (low endemic setting), the authors found strong correlation of positive IGRA in presumed ocular TB. [31] They also found retinal vasculitis and posterior uveitis to be the clinical phenotypes associated with tubercular uveitis (TAU) or ocular TB. Cordero-Coma et al. established immune response prevalence of $32.25 \%$ in 31 uveitis patients from Spain with estimated sensitivity of $82 \%$ and specificity of $100 \%$ with NPV of $86 \%$ for positive QFT. [32] Mackensen et al. in 2008 and Gupta et al. in 2010 had established the association of QFT with serpiginous like choroiditis. $[6,33]$ Babu et al. have demonstrated the use of QFT in the diagnosis of presumed ocular TB in a prospective pilot study from a high endemic region. [34] Sensitivity of QFT was shown to be $82 \%$ with specificity of $76 \%$ for diagnosis of intraocular TB based on clinical signs suggested by Gupta et al. [6, 34] However, in patients with a definite culture or biopsy 
proven systemic $\mathrm{TB}$, the test has shown only $58 \%$ sensitivity and $77 \%$ specificity, highlighting the limitation of QFT in diagnosing active systemic TB [34].

In a population where $\mathrm{TB}$ is non-endemic, such as the UK, sensitivity of IGRAs is thought not to be as good as TST. [35, 36] Furthermore, specificity of the test has also been questioned. If IGRAs have helped clinicians with a new window of opportunity to diagnose latent $\mathrm{TB}$ it has also caused a reasonable degree of confusion with regards to its false positive and false negative results, with particular variation in low-endemic regions. It is hence essential to review the application and utility of IGRAs in low-endemic countries such as the UK.

The use of ATT to manage ocular TB is regarded as an effective treatment for ocular $\mathrm{TB}$ and response to therapy can be a good surrogate for diagnosis of ocular TB. Bansal et al. showed a reduced recurrence of ocular TB following ATT, in addition to steroids. [37] ATT clears latent $\mathrm{TB}$ infection and subsequently protects against the manifestation of active TB in up to $90 \%$ of patients. [38] Overall it would be expected that ATT would have been effective in treating ocular TB in this study and thus preventing any recurrence. It is unlikely that treatment failure in this study group could be attributed to failure of the ATT itself, and it can be compounded due to the heterogeneity in treatment regimen, inadequate treatment length, antibiotic resistance or non-compliance with the drug regimen.

Based on our observation, it can be postulated that patients with suspected ocular TB get started on steroids early if symptoms are more severe, and for the same reason they are more likely to really have ocular TB hence higher QFT. With past history of pulmonary TB, patients are enrolled in the dataset as suspected ocular TB. As the current study was not a trial of ATT and attempting to analyse the data in terms of treatment efficacy runs the risk of obtaining falsely significant results by multiple testing and confounding by indication. We sought to explore patterns in the data and generate rather than test hypotheses. Likewise, the effect of response to therapy in patients with peripheral retinal vasculitis following panretinal laser photocoagulation could not be assessed in this series, but we have highlighted the outcome of treatment in patients with peripheral retinal vasculitis in our recently published report [39].

This study has several limitations as data was collected retrospectively and researchers have made a decision based on the facts present at their disposal, which can introduce investigator's bias. There was no information about proportion of $\mathrm{TB}$ uveitis patients (amongst all the new uveitis cases seen in a given year), about how many patients had new episodes of inflammation and how many had old ongoing inflammation. There was also a significant variation between oral corticosteroids, immunosuppression and ATT treatment type and length prohibiting us from drawing definitive conclusions. The definition of treatment outcome requires further validation as there is currently no gold standard or consensus guidelines defining the treatment outcome in patients with ocular TB. Furthermore, as the study was from a single centre, it may be difficult to generalize the results of this study. We propose that future directions should include conducting prospective well-designed multicentre (between low and high endemic regions) studies to understand the true formulate and accurate global depiction global picture of presumed ocular TB. A carefully designed multicentre study with clear case definition for ocular TB, non ambigous treatment outcomes, defined follow up with standardized therapy (if not randomized control trial) can tell us more information and perhaps lead to a definite conclusion.

\section{Conclusions}

None of the defined clinical phenotypes of ocular TB or positive QFT were associated with favourable outcome with ATT. There was no evidence for a cut-off value of QFT that successfully discriminated between the two groups of patients. Extrapulmonary mycobacterium TB manifesting in the eye remains a diagnostic conundrum.

\section{Additional files}

Additional file 1: Technical appendix to "Does IGRA test add to the diagnosis of presumed ocular tuberculosis - A Bayesian latent class analysis". (DOCX 52 kb)

Additional file 2: The dataset. (R $25 \mathrm{~kb})$

\section{Abbreviations}

ACE: Angiotensin converting enzyme; ATT: Anti tubercular therapy; AUC: Area under curve; IGRA: Interferon gamma release assay; IL2: Interleukin -2; M: Mycobacterium; NPV: Negative predictive value; PCR: Polymerase chain reaction; PPV: Positive predictive value;

QFT: Quantiferon -TB gold in tube test; ROC: Receiver operating characteristics; TAU: Tubercular associated uveitis; TB: Tuberculosis; TNF a: Tumor necrosis factor; TST: Tuberculin skin test; UK: United Kingdom

\section{Acknowledgements}

This work was supported by the National Institute for Health Research (NIHR) Biomedical Research Centre based at Moorfields Eye Hospital NHS Foundation Trust and UCL Institute of Ophthalmology. The views expressed are those of the author(s) and not necessarily those of the NHS, the NIHR or the Department of Health. The authors would like to acknowledge support from Margaret Richards who helped in identifying case records with QFT at our centre. The authors will also acknowledge support from Dr. Farzana Rahman, Dr. Ioanna Triantafyllopoulou and Dr. Sumita Phatak for their assistance with the data collection.

\section{Funding}

"No specific funding was received for this study".

Availability of data and materials

The study team has the entire data and data can be shared with the publisher/ editorial team/ reviewers as and when required. Please contact authors for data requests. 


\section{Authors' contributions}

RA designed and conceptulaised the study. RG did statistical analysis and wrote major part of the manuscript. BG, DG, JJGL participated in editing the manuscript and writing portions of the manuscript. PA and MW contributed the cases and data for the study and also provided inputs for the manuscript. CP designed and conceptualised the study and contributed the patients for the study. "All authors have read and approved the final version of this manuscript".

\section{Ethics approval and consent to participate}

Moorfields Eye Hospital Research and Ethics Committee Approved the study. Study involved human participants and human data. It was a retrospective study and hence the consent from the patients was waived under this study Study ethics number: ROAD/14/012.

\section{Consent for publication}

Not applicable

\section{Competing interests}

JJGL has received study grants from Alcon, Novartis and Merck, and has provided unpaid consultancy to Bayer. RA was on NMRC overseas research training fellowship at Institute of Ophthalmology and Moorfields Eye Hospital, London. There are no competing interests for any of the authors in this study and there is no funding for this project.

\section{Publisher's Note}

Springer Nature remains neutral with regard to jurisdictional claims in published maps and institutional affiliations.

\section{Author details \\ ${ }^{1}$ Moorfields Eye Hospital, NHS Foundation Trust, London, UK. ${ }^{2}$ Biomedical Research Centre, UCL Institute of Ophthalmology, London, UK. ${ }^{3}$ National Healthcare Group Eye Institute, Tan Tock Seng Hospital, Tan Tock Seng, Singapore. ${ }^{4}$ St George's, University of London \& Kingston University, London, UK. ${ }^{5}$ Royal Berkshire Hospital NHS Foundation Trust, Reading, UK.}

\section{Received: 13 April 2017 Accepted: 5 November 2017} Published online: 08 December 2017

\section{References}

1. Annual TB Update. In: England PH, editor. England. 2014;2014:1-12.

2. Abouammoh M, Abu El-Asrar AM. Imaging in the diagnosis and management of ocular tuberculosis. Int Ophthalmol Clin. 2012;52:97-112.

3. Gupta B, Agrawal R, Swampillai AJ, Lim R, Kee A, Gunasekeran D, Pavesio CE. Ocular manifestations of tuberculosis: an update. Expert Rev Ophthalmol. 2016;11:10-30

4. Gupta V, Gupta A, Rao NA. Intraocular tuberculosis-an update. Surv Ophthalmol. 2007;52:561-87.

5. Gupta A, Sharma A, Bansal R, Sharma K. Classification of intraocular tuberculosis. Ocul Immunol Inflamm. 2015;23:7-13.

6. Gupta A, Bansal R, Gupta V, Sharma A, Bambery P. Ocular signs predictive of tubercular uveitis. Am J Ophthalmol. 2009;149:562-70.

7. Gupta A, Gupta V. Tubercular posterior uveitis. Int Ophthalmol Clin. 2005; 2005(45):71-88.

8. Garip A, Diedrichs-Mohring M, Thurau SR, Deeg CA, Wildner G. Uveitis in a patient treated with Bacille-Calmette-Guerin: possible antigenic mimicry of mycobacterial and retinal antigens. Ophthalmol. 2009;116:2457-62.

9. Ang M, Wong WL, Kiew SY, Li X, Chee SP. Prospective head-to-head study comparing 2 commercial interferon gamma release assays for the diagnosis of tuberculous uveitis. Am J Ophthalmol. 2014;157:1306-14.

10. Kleinert S, Kurzai O, Elias J, Marten K, Engelke C, Feuchtenberger M, Sandstede J, Forsch M, Tony HP, Kneitz C. Comparison of two interferongamma release assays and tuberculin skin test for detecting latent tuberculosis in patients with immune-mediated inflammatory diseases. Ann Rheum Dis. 2010;69:782-4.

11. Ang M, Htoon HM, Chee SP. Diagnosis of tuberculous uveitis: clinical application of an interferon-gamma release assay. Ophthalmol. 2009; 116:1391-6

12. MMWR. Recommendations and reports. Morbidity and mortality weekly report. Recomm Rep / Centers Dis Control. 2006;55(RR-9):9.
13. Gineys R, Bodaghi B, Carcelain G, Cassoux N, le TH B, Amoura Z, Lehoang P, Trad S. QuantiFERON-TB gold cut-off value: implications for the management of tuberculosis-related ocular inflammation. Am J Ophthalmol. 2011;152:433-40.

14. Ongchin S, Keene CD, Van Gelder R, Vemulakonda GA. A diagnostic dilemma: infectious versus noninfectious multifocal choroiditis with panuveitis. J Ophthalmic Inflamm Infect. 2013;3(1):26.

15. Brownell I, Ramírez-Valle F, Sanchez M, Prystowsky S. Evidence for mycobacteria in sarcoidosis. Am J Respir Cell Mol Biol. 2011;45:899-905.

16. Ang $M$, Wong WL, Li $X$, Chee SP. Interferon $y$ release assay for the diagnosis of uveitis associated with tuberculosis: a Bayesian evaluation in the absence of a gold standard. Br J Ophthalmol. 2013;97:1062-7.

17. Menzies D, Pai M, Comstock G. Meta-analysis: new tests for the diagnosis of latent tuberculosis infection: areas of uncertainty and recommendations for research. Ann Intern Med. 2007:146:340-54. Erratum in (2007): Annals of Internal Medicine 146:688

18. Urzua CA, Liberman P, Abuauad S, Sabat P, Castiglione E, Beltran-Videla MA Aguilera R. Evaluation of the accuracy of T-SPOT.TB for the diagnosis of ocular tuberculosis in a BCG-vaccinated, non-endemic population. Ocul Immunol Inflamm. 2016;4:1-5.

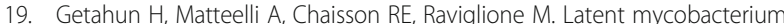
tuberculosis infection. N Engl J Med. 2015;372:2127-35.

20. Rosenbaum JT, Wernick $R$. The utility of routine screening of patients with uveitis for systemic lupus erythematosus or tuberculosis- a Bayesian analysis. Arch Ophthalmol. 1990;108:1291-3.

21. Quantiferon TB-Gold EllSA Package insert (Qiagen, Australia) (2013). Website URL: http://www.hshc.com.tw/data/files/201612/o 1b2vd080mcmg16nh1c3gg8v1hsd9.pdf. Accessed 19 Nov 2017.

22. Altman DG. Practical statistics for medical research. USA: CRC Press; 1990.

23. Agrawal R, Kee AR, Ang L, Tun Hang Y, Gupta $V$, Kon OM, Mitchell D, Zierhut M, Pavesio C. Tuberculosis or sarcoidosis: opposite ends of the same disease spectrum? Tuberculosis (Edinb). 2017:98:21-6.

24. Lee S-Y, Song X-Y. Basic and advanced Bayesian structural equation modeling: with applications in the medical and behavioral sciences. Hoboken: Wiley; 2012

25. Van Smeden M, Naaktgeboren CA, Reitsma JB, Moons KGM, de Groot JAH. Latent class models in diagnostic studies when there is no reference standard - a systematic review. Am J Epidemiol. 2014;179:423-31.

26. Gelman A, Carlin JB, Stern HS, Dunson DB, Vehtari A, Rubin DB. Bayesian data analysis: Third edition. Boca Raton: Chapman \& Hall; 2013.

27. Ang M, Cheung G, Vania M, Chen J, Yang H, Li J, Chee SP. Aqueous cytokine and chemokine analysis in uveitis associated with tuberculosis. Mol Vis. 2012;18:565-73

28. Thayil SM, Albini TA, Nazari H, Moshfeghi AA, Parel JM, Rao NA, Karkousis PC. Local ischemia and increased expression of vascular endothelial growth factor following ocular dissemination of mycobacterium tuberculosis. PLoS One. 2011:6:e28383.

29. La Distia NR, van Velthoven ME, Ten Dam-van Loon NH, Misotten T, Bakker M, van Hagen MP, Rothova A. Clinical manifestations of patients with intraocular inflammation and positive QuantiFERON-TB gold in-tube test in a country nonendemic for tuberculosis. Am J Ophthalmol. 2014:157:754-61.

30. Pepple KL, Van Gelder R, Forooghian F. Caveats about QuantiFERON-TB gold in-tube testing for uveitis. Am J Ophthalmol. 2014;157:752-3.

31. Ahn SJ, Kim KE, Woo SJ, Park KH. The usefulness of interferon-gamma release assay for diagnosis of tuberculosis-related uveitis in Korea. Korean J Ophthalmol. 2014;28:226-33.

32. Cordero-Coma M, Calleja S, Torres HE, del Barrio I, Franco M, Yilmaz T, Vivas $\mathrm{S}$, de Morales JG. The value of an immune response to mycobacterium tuberculosis in patients with chronic posterior uveitis revisited: utility of the new IGRAs. Eye. 2010;24:36-43.

33. Mackensen F, Becker MD, Wiehler U, Max R, Dalpke A, Zimmermann S. QuantiFERON TB-gold-a new test strengthening long-suspected tuberculous involvement in serpiginous-like choroiditis. Am J Ophthalmol. 2008;146:761-6.

34. Babu K, Satish V, Satish S, Subbakrishna DK, Abraham MP, Murthy KR. Utility of QuantiFERON TB gold test in a south Indian patient population of ocular inflammation. Ind J Ophthalmol. 2009:57:427-30.

35. Albini TA, Karakousis PC, Rao NA. Interferon-gamma release assays in the diagnosis of tuberculous uveitis. Am J Ophthalmol. 2008;146:486-8.

36. Ang $M$, Wong $W L$, Li $X$, Chee SP. Interferon gamma release assay for the diagnosis of uveitis associated with tuberculosis: a Bayesian evaluation in the absence of a gold standard. Br J Ophthalmol. 2013;97:1062-7. 
37. Bansal R, Gupta A, Gupta V, Dogra MR, Bambery P, Arora SK. Role of antitubercular therapy in uveitis with latent/manifest tuberculosis. Am J Ophthalmol. 2008;146:772-9.

38. American Thoracic Society. Targeted tuberculin testing and treatment of latent tuberculosis infection. MMWR Recomm Rep. 2000;49(RR-6):1-51.

39. Agrawal R, Gunasekeran DV, Gonzalez-Lopez JJ, Cardoso J, Gupta B, Addison PK, Westcott M, Pavesio CE. Peripheral retinal vasculitis: analysis of 110 consecutive cases and a contemporary reappraisal of tubercular etiology. Retina. 2017;37(1):112-7.

Submit your next manuscript to BioMed Central and we will help you at every step:

- We accept pre-submission inquiries

- Our selector tool helps you to find the most relevant journal

- We provide round the clock customer support

- Convenient online submission

- Thorough peer review

- Inclusion in PubMed and all major indexing services

- Maximum visibility for your research

Submit your manuscript at www.biomedcentral.com/submit 